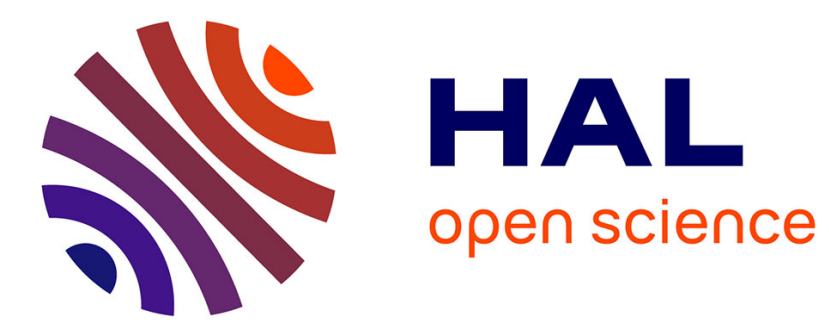

\title{
Biological organization and cross-generation functions
} Cristian Saborido, Matteo Mossio, Alvaro Moreno Bergareche

\section{To cite this version:}

Cristian Saborido, Matteo Mossio, Alvaro Moreno Bergareche. Biological organization and crossgeneration functions. British Journal for the Philosophy of Science, 2011, 62 (3), pp.583-606. 10.1093/bjps/axq034 . halshs-00792437

\section{HAL Id: halshs-00792437 \\ https://shs.hal.science/halshs-00792437}

Submitted on 30 Mar 2015

HAL is a multi-disciplinary open access archive for the deposit and dissemination of scientific research documents, whether they are published or not. The documents may come from teaching and research institutions in France or abroad, or from public or private research centers.
L'archive ouverte pluridisciplinaire HAL, est destinée au dépôt et à la diffusion de documents scientifiques de niveau recherche, publiés ou non, émanant des établissements d'enseignement et de recherche français ou étrangers, des laboratoires publics ou privés.

\section{()ㅜ(1)}

Distributed under a Creative Commons Attribution - NonCommercial| 4.0 International 
BIOLOGICAL ORGANIZATION

AND CROSS-GENERATION FUNCTIONS ${ }^{1}$

Br J Philos Sci (2011) 62 (3): 583-606

http://dx.doi.org/10.1093/bjps/axq034
Cristian Saborido Department of Logic and Philosophy of Science, University of the Basque Country, Avenida de Tolosa 70, 20080, San Sebastian, Spain

Matteo Mossio Institut d'Histoire et de Philosophie des Sciences et des Techniques, CNRS/Université Paris1/ENS, 13 rue du Four, 75006, Paris, France

$\&$

Department of Logic and Philosophy of Science, University of the Basque Country, Avenida de Tolosa 70, 20080, San Sebastian, Spain

\begin{abstract}
Alvaro Moreno Department of Logic and Philosophy of Science, University of the Basque Country, Avenida de Tolosa 70, 20080, San Sebastian, Spain
\end{abstract}

\begin{abstract}
The organizational account of biological functions interprets functions as contributions of a trait to the maintenance of the organization that, in turn, maintains the trait. As it has been recently argued, however, the account seems unable to provide a unified grounding for both intra-generation and cross-generation functions, since the latter do not contribute to the maintenance of the same organization which produces them. To face this "ontological problem", a splitting account has been proposed, according to which the two kinds of functions require distinct organizational definitions. In this paper, we propose a solution for the ontological problem, by arguing that intra- and cross-generation functions can be said to contribute in the same way to the maintenance of the biological organization, characterized in terms of organizational self-maintenance. As a consequence, we suggest maintaining a unified organizational account of biological functions.
\end{abstract}

\footnotetext{
${ }^{1}$ The work was funded by Ministerio de Ciencia e Innovación, Spain ('Juan de la Cierva' program to MM); Ministerio de Ciencia e Innovación, (grant no. FFI2008-06348-C0201/FISO \& FFI2008-06348-C02-02/FISO and BFU2006-01951/BMC); Gobierno Vasco (predoctoral fellowship to CS and grant no. 937 IT-250-07); and the European Union (Feder funds).
} 


\section{Introduction}

In recent years, the philosophical debate on the concept of biological function has been enriched by the development of a new approach, aimed at integrating the two mainstream views - the 'etiological' (Wright, 1973; Millikan, 1984, 1989; Neander, 1980, 1991; Godfrey-Smith, 1994) and the 'systemic-dispositional' (Cummins, 1975; Boorse, 1976; Bigelow \& Pargetter, 1987) - into an inclusive framework. The gist of the approach consists of relating functions to a distinctive feature of biological systems, i.e. the fact that they realize a specific kind of causal regime in which a set of parts generates a complex web of mutual interactions which, in turn, maintains the parts, so that the whole organization is collectively able to maintain itself through time. In what follows, we will refer to this specific causal regime as a self-maintaining organization.

Within a self-maintaining organization, in particular, functions are interpreted as specific causal effects of a part or trait, which contribute to the maintenance of the organization and, consequently, of the part itself. As has been emphasized (Schlosser, 1998: 327-328; Mossio et al., 2009: 1213), this characterization also offers a way of naturalizing into the current organization of biological systems the two characteristic properties of functions which call for a scientifically acceptable explanation, and which have given rise to the long-lasting philosophical debate on this notion, i.e. their teleology and normativity.

On the one hand, a self-maintaining organization provides a naturalized grounding for the teleological dimension. Since the activity of a biological component $\mathrm{X}$ contributes, by contributing to the maintenance of the whole organization, to the maintenance of some of the conditions required for its own existence, the question "Why does $\mathrm{X}$ exist in that system?" can be legitimately answered by "Because it does Y". This justifies explaining, "teleologically", the existence of a component by referring to its causal effects. On the other hand, a self-maintaining organization grounds normativity. The activity of a self-maintaining organization has an intrinsic relevance for itself, to the extent that its very existence depends on the effects of its own activity. Such intrinsic relevance generates a naturalized criterion to determine what norms the system, and its parts, are supposed to follow. The whole system (and its parts) must behave in a specific way, otherwise it would cease to exist. Accordingly, the activity of the system and its components becomes its own norm or, more precisely, the conditions of existence for the organization are the intrinsic and naturalized norms of its own activity and of that of its components ${ }^{\mathrm{ii}}$.

Because of its appeal to fundamental properties of biological organization, we have suggested labeling this account the organizational account to functions $^{\text {iii }}$, on which a considerable amount of work has already been done by Bickhard (2000, 2004), Schlosser (1998), Collier (1999), McLaughlin (2001), Christensen \& Bickhard (2002) Delancey (2006), Edin (2008) and Mossio et al. (2009). In spite of some differences between the various formulations, there seems to be substantial convergence regarding the fundamental tenets of the organizational account, which makes it a credible philosophical alternative to both the etiological (mainly in its "selected- 
effects" version) and systemic-dispositional accounts. In a recent analysis, however, Craig Delancey (2006) emphasized a major theoretical challenge facing the organizational account. This challenge can be described as follows.

Since functions are characterized as contributions of parts to selfmaintaining organizations, and since self-maintaining organizations are typically realized by individual organisms, the organizational approach appears to have trouble grounding functions which go beyond the boundaries of individual biological systems. In particular, it is unclear whether and, indeed, how the organizational approach would account for what Schlosser calls "cross-generation functions", for instance the function of reproductive traits (e.g. the function of semen of inseminating the ovum). In these cases, in fact, the trait or part seems to contribute to the maintenance of the organization of a system which is distinct from the system of which it is a component. Hence, the part does not contribute either to the self-maintenance of an organization or to its own selfmaintenance. Still, we do ascribe cross-generation functions as we do, for instance, for the reproductive function of the semen. Apparently, then, cross-generation functions may constitute a major class of counterexamples of the organizational approach.

Some of the authors who developed previous versions of the organizational account were of course aware of this issue, and proposed - following very different paths, as we shall see - definitions which were supposedly broad enough to embrace both intra- and cross-generation biological functions. Nevertheless, Delancey's analysis criticizes all these "unified accounts" by pointing out their weaknesses and drawbacks. As an alternative, he proposes a "splitting account", according to which intra-generation and crossgeneration functions are in fact two different kinds of biological function, requiring a different conceptual treatment.

The aim of this paper is to offer a philosophical reply to Delancey's criticism. By relying on the conceptual framework that we recently proposed (Mossio et al., 2009), we will suggest that the organizational account may provide a unified definition applying to both intra-generation and cross-generation functions. The essence of our argument will be that cross-generation functions contribute to the maintenance of systems which realize a self-maintaining organization in the very same sense as that of systems whose parts are ascribed intra-generational functions. To the extent that the two kinds of systems do not differ in terms of organizational selfmaintenance, there is no need to invoke two kinds of functions, and the ontological problem is overcome. By showing that a unified organizational account of function can be justified, we suggest applying Ockham's razor to this specific case: since pluralitas no es ponenda sine necessitate, there is no need to adopt a splitting account for organizational functions.

The structure of the paper is as follows. In section 2, we will discuss Schlosser's approach, as well as McLaughlin's one, both of which develop a unified account of functions based on the idea of traits' capacity for selfreproduction. We will then examine Delancey's criticism, and recall its proposal of a splitting account. Section 3 will develop our own account of 
cross-generation functions, which emphasizes, instead of the selfreproduction of the trait, the self-maintenance of the whole organization as the relevant dimension in which cross-generation functions can be adequately naturalized. In the conclusion, we will sum up by underlying the reasons why our own framework appears to be in a better position to account for cross-generation function with respect to other organizational theories.

\section{Self-reproduction of traits: proposals and criticism}

Broadly speaking, the existing formulations of the organizational account can be regrouped into two main versions. The first version, advocated by Schlosser (1998) and McLaughlin (2001), tends to characterize functions as states or processes which are causally required for the reproduction of the trait which causes them. The emphasis is then on the self-reproduction of the trait, rather than specifically on the whole system which, nevertheless, must possess the adequate properties to enable trait self-reproduction. The second version, proposed by Collier (1999), Christensen \& Bickhard (2002), and Mossio et al. (2009), shifts the focus onto the organization of the system, and interprets functions as contributions to the self-maintenance of the organization and therefore, as a consequence, to the self-maintenance of the various parts and traits. The two versions give an equivalent response for most function ascriptions, but cases do exist in which they may diverge. Indeed, the first version may have problematic implications for cases in which a trait self-reproduction is in conflict with the self-maintenance of the whole system such as, for instance, in cancer development. In this case, the first version could be forced to attribute, against intuition and scientific use, a function to the trait which self-develops, whereas the second version could unambiguously exclude function ascription to cancerous traits. In this paper, we will not analyze the differences and divergences between the two versions in general, but rather will examine specifically how they account for the ascription of cross-generation functions, and we will discuss why, in our view, the second version is in a better position to provide an adequate solution for Delancey's criticism.

\subsection{Schlosser's and McLaughlin's accounts}

Schlosser (1998) proposed ascribing functions to those traits which, in a particular class of complex system and under certain conditions, are selfreproducing. A trait (or state) $\mathrm{X}$ is functional in a given complex system if it causes the process or state $F$ (its function) which, under certain conditions, is necessary for the self-reproduction of $\mathrm{X}$. In this sense, the "functionality of a state can be defined as the conditional necessity for complex self-reproduction (i.e., the necessity for self-reproduction in complex systems under certain circumstances)" (Schlosser, 1998: 315). Systems harboring self-reproducing traits are, in turn, "systems that pass through cyclic sequences of states and thereby keep stable in the long run, despite changing continuously" (Schlosser, 1998: 311). The paradigmatic case of selfreproducing complex systems, to the traits of which functions are ascribable, is that of biological organisms. Schlosser proposes the following definition of functions: 
"In self-re-producing systems that remain stable for a certain period of time $\mathrm{T},[\ldots] \mathrm{F}$ is a function of $\mathrm{X}(\mathrm{t})$ iff:

for a certain period of time $\mathrm{t}_{0}<\mathrm{t}<\mathrm{t}+\mathrm{x}+\mathrm{y}<\mathrm{t}_{0}+\mathrm{T}^{\mathrm{iv}}$

(1) $\mathrm{X}(\mathrm{t})$ is directly causally necessary to establish $\mathrm{F}(\mathrm{t}+\mathrm{x}$ ) (under certain circumstances $\mathrm{c} 1$ )

(2) $F(t+x)$ is indirectly causally necessary to establish $X(t+x+y)$ (under certain circumstances $\mathrm{c} 2$ )

(3) The causal relations between $\mathrm{X}(\mathrm{t}), \mathrm{F}(\mathrm{t}+\mathrm{x}), \mathrm{X}(\mathrm{t}+\mathrm{x}+\mathrm{y})$ are complex" (Schlosser, 1998: 315).

This definition clarifies that the relations between $\mathrm{X}$ and $\mathrm{F}$ are not symmetrical, in the sense that whereas $\mathrm{X}$ causes $\mathrm{F}$ in a "relatively direct way", F causes the re-production of X quite indirectly, usually through the complex web of interactions of the whole system. In addition, (3) restricts functional attributions to a specific class of systems in which selfreproduction of traits occurs, namely "complex systems", in order to exclude trivial counterexamples.

It is worth noting, for the purposes of this paper, that this definition embraces self-reproduction both as the continuous re-establishment of a trait in the same system, and as the re-establishment of a trait of the same type in a new system. For instance, the function of the heart is to pump blood because (1) in a given period of time the heart is directly causally necessary to establish the pumping of the blood and (2) the pumping of the blood is indirectly causally necessary to re-establish the heart, and the human organism is certainly a complex system. Similarly, the function of semen is to inseminate the ovum since (1) the semen is directly causally necessary to establish the insemination of the ovum, (2) the insemination of the ovum is indirectly causally necessary to re-establish the semen (in this case, in a new organism) and (3) the causal relations between the semen, the insemination of the ovum and the new semen are complex.

Clearly, the scheme applies to both intra-generation and cross-generation functions, although the two cases are not equivalent. Whereas the same instance of the trait self-reproduces in the case of intra-generation functions, it is a new instance of the same type that is reproduced through crossgeneration functions ${ }^{\mathrm{v}}$. In Schlosser's framework, this difference is negligible from the point of view of the organizational account, since what matters is the fact that the trait type achieves self-reproduction. Whether this occurs as the re-establishment of the same instance or the establishment of a new instance is seen as irrelevant from the point of view of the account.

In his recent book (2001), Peter McLaughlin develops an organizational account of functions which is similar to Schlosser's one. According to McLaughlin, claiming that the function of $\mathrm{X}$ is $\mathrm{F}$, means that:

“(1) X does/enables F (in or for some system S);

(2) F is good for some $S$;

and

(3) By being good for some S, F contributes to the (re)production of 
$\mathrm{X}$ (there is a feed-back mechanism involving F's benefiting $\mathrm{S}$ that (re)produces X). (McLaughlin 2001: 140)"

In McLaughlin's view, functions are ascribed to parts of systems being, in his terms "appropriate subject of benefit". A system having functional parts is a system having a good. In turn, McLaughlin intimately associates the concepts of good and that of self-reproduction: "If the characteristic activity of an organism is its self-reproduction, then 'good for the characteristic activity of $\mathrm{X}^{\prime}$ and 'good for $\mathrm{X}^{\prime}$ are the same. This is what makes organisms, as opposed to inanimate objects, the appropriate subjects of benefit or harm. It is the fact that 'what they do makes them what they are' - not the (derivative) fact that they are also alive - that occasions us to attribute a good to them. Even a non-organic self-reproducing system could be the subject of benefit and thus have a good" (McLaughlin, 2001: 203).

As we mentioned in the introduction, and will further develop in section 3, all organizational approaches, besides minor differences, adopt this line of thought to justify a naturalized grounding of norms.

McLaughlin emphasizes the causal role of a function in (re)producing the function bearer by appealing to a "feedback mechanism" at work in the system. While the formulation differs from Schlosser's one in various respects, the central idea is very much the same: the function of a trait is its causal effect involved in promoting its own existence. Much like Schlosser, McLaughlin interprets the term "reproduction" in a quite broad way, since the reproduction of a trait X can occur within the same system or in a new and distinct system (McLaughlin 2001: 180-181). Accordingly, traits bearing cross-generation functions contribute, through their function, to their own reproduction, which in this case consists of the production of an organ of the same type in a new individual system.

Both Schlosser's and McLaughlin's accounts of cross-generation functions rely on the idea that the notion of "trait self-reproduction" can be adequately applied to the same trait token in a given system, as well as to another trait token of the same type in a different system. As we will discuss in the following section, however, Delancey has argued that the difference cannot be neglected, and this in turn entails a major revision of the organizational account.

\subsection{Delancey's "splitting account”}

In a recent paper, Craig Delancey (2006) argues that this version of the organizational account suffers from what he calls an "ontological" problem, to the extent that the complex causal relations between the traits and their functions, as they are expressed by (3) in Schlosser's scheme, are radically different depending on whether the functions are intra-generation or crossgeneration. According to Delancey, one reason why Schlosser's account seems to be applicable to both classes of functions is because it does not refer to complex concrete systems as the relevant support for functional ascriptions, but simply to a web of complex relations, such that $\mathrm{X}(\mathrm{t})$ and $\mathrm{X}(\mathrm{t}+\mathrm{x}+\mathrm{y})$ could equally refer to either intra-generation or cross-generation instances of a trait, without modifying the definition.

Yet, Delancey's criticism consists in arguing that the mere reference to 
"complex relations" is too abstract, and underdetermines the grounding of functional ascriptions. In fact, in all real cases, (biological) functions are ascribed to parts or states of biological organisms, in which evident ontological and causal differences exist between intra-generation functions, which concern a single trait in a single system, and cross-generation functions, which reproduce a trait type in different individuals. According to Delancey, then, Schlosser's account succeeds in providing a unified definition of intra- and cross-generation functions by artificially hiding, without adequate justification, relevant distinctions between the two classes of functions ${ }^{\mathrm{vi}}$.

On the basis of this criticism, Delancey develops his own account, which shares with Schlosser's account the idea that the function of a trait consists of its contribution to its own self-reproduction. At the same time, he reformulates Schlosser's scheme by proposing a double characterization of biological functions, which takes into account a distinction between selfsustaining and self-reproducing functions:

" $\mathrm{F}$ is a self-sustaining teleofunction of structure type $\mathrm{S}$ if, for a certain period of time $\mathrm{T}$, and where $\mathrm{t} 1<\mathrm{t} 2<\mathrm{t} 3$, and $\mathrm{T}$ ranges from before $\mathrm{t} 1$ to after $\mathrm{t} 3$ :

1. There is an instance of $\mathrm{S}, \mathrm{s}(\mathrm{t} 1)$, directly causally necessary to establish $\mathrm{F}(\mathrm{t} 2)$ under circumstances $\mathrm{c} 1$.

2. $\mathrm{F}(\mathrm{t} 2)$ is indirectly causally necessary to sustain $\mathrm{s}(\mathrm{t} 3)$ under circumstances c2.

3. The causal relations between $\mathrm{s}(\mathrm{t} 1), \mathrm{F}(\mathrm{t} 2)$, and $\mathrm{s}(\mathrm{t} 3)$ are part of a single complex system.

$\mathrm{F}$ is a self-reproducing teleofunction of structure type $\mathrm{S}$ if, for a certain period of time $\mathrm{T}$, and where $\mathrm{t} 1<\mathrm{t} 2<\mathrm{t} 3$; and where $\mathrm{T}$ ranges from before $\mathrm{t} 1$ to after $\mathrm{t} 3$ :

1. There is an instance of $\mathrm{S}, \mathrm{s} 1(\mathrm{t} 1)$, that is directly causally necessary to establish $\mathrm{F}(\mathrm{t} 2)$ under circumstances $\mathrm{c} 1$.

2. $\mathrm{F}(\mathrm{t} 2)$ is indirectly causally necessary to establish some instance of $\mathrm{S}$, $\mathrm{s} 2(\mathrm{t} 3)$ under circumstances $\mathrm{c} 2$.

3. The causal relations between $\mathrm{s} 1(\mathrm{t} 1), \mathrm{F}(\mathrm{t} 2)$, and $\mathrm{s} 2(\mathrm{t} 3)$ are part of at least one complex system; if they are part of more than one complex system, then these are of the same type of complex system.

4. It is not the case that $\mathrm{s} 1=\mathrm{s} 2$ (Delancey, 2006: 91-92)".

In this scheme then, "complex system" means "individual organism", with no reference to any other broader or encompassing systems. Of course, other systems than individual organisms could be interpreted as complex systems, which would be relevant for function ascriptions. Yet, as Delancey suggests, not only is there no proof that functions could be ascribed to other systems than organisms, but in fact there is no need. Indeed, the distinction between intra- and cross-generation functions is captured by point 4 in the definition of self-reproducing functions, which explicitly states that the 
reproduction concerns a different instance of a trait type.

Delancey's analysis and splitting account emphasizes the difficulties involved in formulating a unified definition able to deal coherently with both intra- and cross-generation biological functions within the organizational view. Delancey himself considers that a unified definition would be, in principle, a more elegant and palatable alternative to his splitting account but, at present, practical reasons suggest adopting his own proposal. As he writes:

"Since the splitting account I describe is sufficient, and is also satisfied by the implicit work that goes into preparing the way for either of the unproblematic forms of the unified account, Ockham's razor cuts in favour of the splitting account alone. This then poses a challenge to the unified account: a viable unified account must explain how we are to identify events or complex relations without reference to individual organisms and their structures; or, it must explain what benefits the account has which make it preferable to the simpler splitting account. If the unified account cannot do either of these, then the splitting account is preferable both because it is simpler and because it is already implicitly satisfied in the preliminary work required to develop a unified account (Delancey, 1996: 94-95)".

We agree with Delancey that a unified definition would be more elegant, and that it should be able to answer Delancey's challenge and demonstrate its superiority over the splitting account. In the following section, we will first introduce our own version of the organizational account and then propose our solution to the ontological problem.

\section{Self-maintenance of systems}

The second main version of the organizational account regroups several contributions developed by Collier (1999), Christensen \& Bickhard (2002), and ourselves (2009) which, despite sharing the same fundamental inspiration as the previous version, follow a different path. Instead of interpreting functions as those effects which contribute to the selfreproduction of the trait within a given kind of complex system, it conceptualizes functions as contributions to the self-maintenance of the system which consequently results in the maintenance of the trait. The various formulations of this second version are basically the same in their core framework, despite some major differences in terminology. However, we believe that our own proposal develops its central concepts and implications in more detail, thus making a comparison with the accounts discussed above easier. For this reason, we will refer to it in the following section.

\subsection{Functions and organizational closure}

In a recent contribution (Mossio et al., 2009), we developed an organizational account of biological functions that relies on the understanding of biological systems as a class of highly complex natural self-maintaining systems. The idea of self-maintenance comes from a theoretical and mathematical framework developed over the last 40 years by 
an increasingly rich body of scientific literature in fields such as theoretical biology, complex systems theory and far-from-equilibrium thermodynamics. This notion refers to a specific causal regime, realized by various kinds of natural systems, by which a given system is able to exert a causal influence on its surroundings so to maintain (at least some of) the boundary conditions required for its own existence.

In its minimal form, the idea of self-maintenance is shown in the so-called "dissipative structures" (Glansdorff and Prigogine, 1971; Nicolis and Prigogine, 1977), i.e. systems in which a macroscopic ordered pattern (a "structure"), emerging in the presence of a specific flow of energy and matter in far-from-thermodynamic equilibrium (FFE) boundary conditions, exerts a constraining action on its boundary conditions that contributes to the maintenance of that FFE flow of energy and matter required for its own persistence (Ruiz-Mirazo, 2001: 59). In nature, a very broad set of physical and chemical systems, such as Bénard cells, flames, whirlwinds, hurricanes and oscillatory chemical reactions can be pertinently described as selfmaintaining dissipative systems (Chandresekhar, 1961; Field et al., 1972; Field and Noyes, 1974).

Since dissipative structures exist only insofar as the adequate boundary farfrom-equilibrium conditions are maintained, and since the structure itself contributes to maintaining these conditions, the activity of the system becomes a necessary (even if not sufficient) condition $-a$ dynamic presupposition, in Bickhard's (2000) terms - for the system itself. The system has to maintain an appropriate interaction with its surroundings to maintain itself.

Our organizational approach claims that self-maintenance constitutes the relevant causal regime in which the teleological and normative dimensions of functions can be adequately naturalized. Let us take the simple example of a flame. The flame, as Bickhard (2000: 114) points out, makes several contributions to the maintenance of the FFE conditions required for its own existence. Indeed, the flame keeps the temperature above the combustion threshold, vaporizes wax, and induces convection (which pulls in oxygen and removes combustion products). Accordingly, to the question "Why does the flame exist?" it is legitimate to answer "Because it does X": the existence of the reactions of combustion (the flame itself) is teleologically explained (at least in part) by taking into account their causal effects.

Moreover, because of the teleological dimension, what a self-maintaining system does is relevant, makes a difference for itself, since its very existence depends on the effects of its activity. Such mutual dependence between their existence and activity, which is specific to self-maintaining systems, provides an intrinsic and naturalized ${ }^{\text {vii }}$ criterion to determine what norms the system, and its parts, are supposed to follow.

The conditions of existence of the system are here interpreted as the norms of its own activity: the flame must behave in a specific way, otherwise it would cease to exist.

While FFE self-maintenance grounds as such the teleological and normative dimensions of functions, it is not enough to naturalize them. Components of dissipative structures do not have functions. The reason is, in our view, that 
the very idea of function requires, in addition to teleology and normativity, that of a certain type of organization based on a set of mutually dependent constraints (Moreno \& Ruiz-Mirazo 2009). Let us develop this idea a bit more. Dissipative structures are minimal self-maintaining systems, in the sense that, although they may be quite complex in terms of the variety of their material components, they produce a single structure that works as a constraint on its own boundary conditions. In dissipative structures, there is only one structure which, by harnessing the microscopic surrounding dynamics, maintains the conditions of its own existence and realizes the teleological and normative dimensions.

In contrast, we believe, functional attributions require an additional dimension, so that the interplay between a set of mutually dependent structures acting as constraints, each of which makes a specific and distinct contribution, realizes self-maintenance by maintaining the boundary conditions at which the whole organization, as well as its various structures, can exist. The mutual dependence between a set of constraints, collectively realizing self-maintenance, is what we call an organizational closure. In organizational closure, each process or part is, to use Bickhard's terms above, dynamically presupposed by the other processes and parts to the overall self-maintenance of the system, such that the whole network must work in a specific and adequate way otherwise, because of its FFE nature, the system would disintegrate ${ }^{\text {viii }}$.

We label the resulting causal regime a self-maintaining organization, and we claim that it constitutes the relevant ground for functional attributions. Indeed, the idea of a self-maintaining organization possesses all the dimensions required to naturalize functions and in particular, in contrast to minimal self-maintaining systems, the internal differentiation of contributions to the maintenance of the system. Since the various constraints exert different causal effects, are mutually dependent and contribute to the maintenance of an organization upon whose maintenance their own existence depends, these parts have functions, and functional ascriptions here play an explanatory role.

The intimate association between complexity and integration at work in a self-maintaining organization is the relevant ground of functional discourse. The most typical example of organizationally self-maintaining systems are biological systems. Consider, for instance, a biological cell. In a cell, different structures make distinct contributions to self-maintenance. The membrane - as a whole, integrated structure made out of many different molecules - acts as a constraint that (among other things) ensures adequate internal concentrations of metabolites. In turn, DNA (among other things) acts as a template for the synthesis of proteins, which have to be continuously renewed due to their high rate of decay. In a word, the cell possesses different parts, produced within and by the system, that contribute differently to the maintenance of the organization and thus, of themselves. In this way, functional attributions to each part are grounded.

Let us recall our definition ${ }^{\mathrm{ix}}$. According to our account, a trait $\mathrm{T}$ has a function if, and only if, it is subject to organizational self-maintenance in a system S. This definition implies the fulfillment of three different 
conditions. Accordingly, a trait $\mathrm{T}$ has a function if and only if:

$\mathrm{C}_{1}$. T contributes to the maintenance of the organization $\mathrm{O}$ of $\mathrm{S}$;

$\mathrm{C}_{2}$. $\mathrm{T}$ is produced and maintained under some constraints exerted by $\mathrm{O}$

$\mathrm{C}_{3}$. S realizes organizational closure.

Accordingly, the heart has the function of pumping blood since $\left(\mathrm{C}_{1}\right)$ pumping blood contributes to the maintenance of the organism by allowing blood to circulate, which in turn enables the transport of nutrients to and waste away from cells, the stabilization of body temperature and $\mathrm{pH}$, and so on. At the same time, $\left(\mathrm{C}_{2}\right)$ the heart is produced and maintained under various constraints exerted by the organism, whose overall integrity is required for the ongoing existence of the heart itself. Lastly $\left(\mathrm{C}_{3}\right)$, the organism realizes organizational closure, since it is constituted by a set of mutually dependent structures which, by contributing in different ways to the maintenance of the organization, collectively realize self-maintenance.

Is our organizational account able to account simultaneously for intra- and cross-generation functions? At first sight, it does not. Cross-generation traits, while they do have a function in biological systems, do not seem to be subject to organizational closure in any individual system. Indeed, crossgeneration traits are not subject to closure in either the reproducing system (in which they satisfy $C_{2}$ but not $C_{1}$ ), since they do not contribute to the selfmaintenance of the system producing and maintaining them, or the reproduced system (in which they satisfy $\mathrm{C}_{1}$ but not $\mathrm{C}_{2}$ ). In this sense, it seems that, in our organizational account, cross-generation traits are not functional with respect to any concrete self-maintaining system.

Should we adopt a splitting account so that, when applied to crossgeneration functions, the instance of the trait $\mathrm{T}$ mentioned in $\mathrm{C}_{1}$ is not the same as that mentioned in $\mathrm{C}_{2}$, even if they belong to the same type? In what follows we will try to show that we do not need to follow that path.

\subsection{Second order self-maintaining systems?}

A possible alternative solution to the splitting account, different from Schlosser's one, has been envisaged by Christensen and Bickhard (2002) who, by developing ideas formulated by Bickhard $(2000 ; 2004)$ and Collier (1999), have proposed an organizational account of functions which is very close to that we described in the previous section. The central idea of this account consists of naturalizing the teleological and normative dimensions of functions through the notion of biological autonomy. Biological systems are autonomous systems in the sense of being self-governed systems, constituted by a network of interdependent processes, and continuously maintained by their own internal organization. In addition, they are able to modify themselves and modulate their behavior in response to external stimulation, in order to maintain or even improve their global viability.

In their view, the very idea of functionality is related to the contribution to the maintenance of the conditions of existence of an autonomous system. Contributing to autonomy is as such functional because the contribution of each component or trait helps, in the end, to contribute to the continued 
existence of the system and therefore, because of the interdependence between the parts, to their own maintenance. "This is the core for the emergence of function: a contribution to the maintenance of the far-fromequilibrium conditions of a far-from-equilibrium system is functional, it serves a function, for the stability, the persistence, of that system" (Bickhard, 2004: 129).

How do they handle cross-generation functions? The proposal tabled by Christensen \& Bickhard (and Collier) consists of explicitly granting systems (like populations or species) the status of autonomous systems, making them relevant supports for functional attributions just as individual organisms: "Living organisms in general are autonomous systems, as are reproductive lineages, species, and some kinds of biological communities' (Christensen \& Bickhard 2002: 3). As a consequence, intra-generation and cross-generation functions are simply contributions to the maintenance of the autonomous organization of different kinds of concrete systems. Whereas intra-generation functions would contribute to the autonomous organization of individual organisms, cross-generation functions would contribute to the autonomous organization of the lineage, the species or the biological community in question.

Christensen \& Bickhard offer an elegant alternative to the splitting account by admitting the idea of "second-order autonomous systems", more complex systems which would include individual organisms as parts, and which would ground the ascription of cross-generation functions. Accordingly, metabolic pathways are functional because they contribute to the autonomy of the cell, the heart is functional because it contributes to the autonomy of the vertebrate, and the semen is functional because it contributes to the autonomy of the species.

Yet, this solution, just as Schlosser's one, is problematic, as Delancey's lucid criticism (2006) shows. As this author points out, the fact of considering those higher-order systems that are relevant for grounding cross-generation functions as autonomous systems does not come without a price. Whereas an individual organism is a paradigmatic case of an autonomous system, "the sense in which the species or some population is a complex system of the appropriate kind is much more difficult to discern" (Delancey, 2006: 90). For instance (the list could be longer), such higherlevel systems have no clear boundaries and no stable form and it is hard to see how their identity is generated by their own internal organization, as is the case for autonomous systems.

According to Delancey, the organizational account has not explored these radical differences with sufficient accuracy, which means that the interpretation of higher-level systems as autonomous systems appears, to say the least, to be an ad hoc hypothesis to cover reluctant cases. To the extent that Christensen \& Bickhard appeal to the idea of autonomy in a fairly general sense, we assume that Delancey's criticism applies equally to an interpretation of higher-level systems as organizational self-maintaining systems, which could be put forward within our own conceptual framework ${ }^{\mathrm{x}}$.

Yet, one might argue that other biological supra-organismic systems do 
possess the properties required to be considered FFE self-maintaining organizations. Let us explore another possibility, not mentioned by Delancey's analysis: the ecosystem. Compared to species, lineages or populations, there seems indeed to be better reasons for considering ecosystems as higher-level systems, relevant for functional attributions, especially if one adopts our characterization in terms of self-maintaining organization, rather than in the more demanding terms of autonomy. Although there are clear differences (just to mention one: the ecosystem has no physical boundaries), ecosystems share several organizational properties with individual organisms. For instance, the various components (many of them being the individual organisms themselves) contribute to maintaining a global organization (the ecosystem itself), which in turn is a general condition for their own continuous existence. Similarly, the various (categories of) components seem to be mutually dependent, so that that the disappearance, death or anomalous behavior of one may provoke the collapse of the whole ecosystem.

For these and other reasons, the ecosystem has much in common with an organism, and in fact it does not seem unreasonable, despite being somewhat uncommon, to use a functional discourse to describe it. So, for instance, we could describe and explain the organization of an ecosystem by attributing to its various components functions such as the regulation of air, climate, water, water supply, disturbance prevention, soil formation and erosion, nutrient cycling, waste treatment, pollination, biological control of pests and diseases and so on (de Groot et al 2002). Specifically, crossgeneration traits would have the function of regenerating the various components of the ecosystem, which would tend to decay because of their dissipative nature.

In our view, the idea that the ecosystem is at least an organizational selfmaintaining system, and possibly an autonomous system, is an attractive one, deserving further investigation. Yet we think that, although a coherent description of whatever second-order system in terms of autonomy or organizational self-maintenance could be provided, the issue would be largely irrelevant for solving the problem of cross-generation functions within the organizational approach. In our view, in fact, the reason why we attribute functions to cross-generation traits is not related to their contribution to the maintenance of some higher-level system. Crossgeneration functions, as we will argue in the following section, do not require a full-fledged account of higher-level autonomous or selfmaintaining systems to be adequately naturalized within an organizational account.

\subsection{A solution of the ontological problem}

The gist of our account of cross-generation functions consists of arguing that the apparent difficulty in integrating them into the definition does not stem from an ontological difference between intra- and cross-generation functions but, rather, from an inadequate understanding of what a selfmaintaining organization actually is. Cross-generation functions constitute a "recalcitrant" class of functions only if the boundaries of the selfmaintaining organization are confused with the boundaries of the individual 
organisms, whereas in fact, as we will argue below, they are not the same. Once this confusion has been cleared up, Delancey's ontological problem disappears.

In our account, functional traits are those traits that, by being submitted to organizational closure, contribute to the maintenance of an organization, which in turn exerts some causal influence on the production and maintenance of the traits. The whole system, as we discussed, realizes a self-maintaining organization. The first remark is that a self-maintaining organization occurs in time, and can be observed only in time. Thus, ascribing functions to traits or parts requires the consideration of a system that realizes self-maintenance during a period of time long enough for organizational closure to be observed. Arguably, the relevant time scale may vary according to the specific function: the organizational closure to which the function of the lung is subject is realized in a very short period of time (one cannot stop breathing for more than a few seconds) whereas, for instance, the function of the stomach is submitted to organizational closure over a longer period of time (one can stop eating for days). Yet, although the period of time may vary, the very idea of organizational closure requires the assumption that the organization whose trait performs a function at time $T_{1}$ is the same organization that maintains the trait at $T_{2}$.

Now, a self-maintaining organization, as an intrinsically temporally extended phenomenon, may undergo various changes and modifications in time. Indeed, as mentioned above, organizational self-maintenance occurs in far-from-equilibrium conditions, which means, among other things, that biological self-maintaining systems (and their parts) are essentially precarious, tend to decay and cannot exist except in the presence of an adequate flow of energy and matter, which enables the continuous regeneration of the whole organization. Self-maintenance occurs, then, in spite of the continuous replacement of the material components. In this sense, a self-maintaining system, be it physical or biological, remains the same while changing all the time, because of its dissipative nature. In addition, biological systems may also undergo mayor structural and morphological changes during their lifetime, due to adaptations, accidental events (injuries, etc.) and, especially, because of development.

If, due to their material, structural and morphological differences, one were to consider the various temporal instances $\mathrm{O}_{1}, \mathrm{O}_{2} \ldots \mathrm{O}_{n}$, as distinct systems with distinct organizations, then self-maintenance, organizational closure and functions could not exist. A trait would be produced by a given organization, let us say $\mathrm{O}_{1}$, and would contribute to maintaining another organization, let us say $\mathrm{O}_{2}$. No organization would actually self-maintain, no trait could be submitted to organizational closure and functions could not be ascribed from an organizational perspective.

The organizational account of functions relies, then, on the theoretical assumption according to which the various temporal instances of a system, in spite of any changes which may occur, can be considered as instances of the same encompassing self-maintaining organization, to the extent that their constitutive organizational properties are causally transmitted from one instance to another instance by the maintenance of a material connection 
between them. The causal transmission of the organization (and specifically its constitutive processes and relations between components) through the various (temporal) instances of a system is the criterion by which the encompassing system, as well as its parts, is described as being the same, and thus, in the case in question here, able to realize self-maintenance although, of course, other criteria could also exist on the basis of which the various instances would not be taken as instances of the same system. If, in a relevant sense, a given system or a given temporal instance of a system possesses a constitutive organization because of its causal and material connection with a previous instance possessing the same organization, then it can be claimed that the two systems or the two instances are in fact instances of the same encompassing organization. In particular, the same is also true if the transmitted organization is a self-maintaining organization.

The central thesis of this paper is that self-maintaining organizations, which ground the ascription of cross-generation functions, and specifically reproductive functions, meet with the very same characterization as those organizations which ground intra-generation functions. While they may differ in important ways, the two classes of self-maintaining organizations do not differ with respect to the relevant properties that ground functional ascriptions.

Cross-generation functions are subject to organizational closure within the frame of self-maintaining organizations whose extension in time goes beyond the lifespan of individual organisms. For instance, the mammal semen, by inseminating the ovum, contributes to the maintenance of the organization by contributing to the production of a new organism to replace the previous one. In turn, the organization (realized by both the reproducer and the reproduced system) exerts several constraints under which the semen is produced and maintained. The crucial point is that the organization of the system constituted by the conjunction of the reproducing and reproduced organisms (in this specific case, a minimal lineage with two elements) has exactly the same status, in terms of self-maintenance, as that of the individual organisms. The fact of considering the organization of individual organisms or their conjunction as the relevant self-maintaining organization is only a question of "zooming", and depends on the explanatory exigencies for functional ascriptions.

Since what matters in the case of organizational self-maintaining systems is the fact that they use their own constitutive organization to exert a causal influence on the maintenance of (at least part of) their own conditions of existence, then the organization of the "encompassing system" composed by a reproducer and a reproduced system itself fits the characterization of a self-maintaining organization. The process of reproduction, in this sense, simply constitutes one of the functions through which the organization succeeds in maintaining itself beyond the lifespan of individual organisms. Since the encompassing system composed by the reproducer and reproduced organism possesses a (temporally wider) self-maintaining organization, reproductive traits are subject to organizational closure, and their functions are correctly grounded in the organizational account.

Moreover, the organization of individual organisms is rather a process of 
becoming - a process of development - followed by a process of decay, than a stable, unchanged, situation of self-maintenance. In the history of each organism, we witness therefore an unfolding of (different forms of) self-maintenance (within a given identity), where reproduction ensures the long-term continuity of a given self-maintaining identity.

Why do cross-generation functions appear problematic? Intuitively, the ontological problem discussed by Delancey seems to apply to our account insofar as reproduction involves a dramatic transition from the reproducer to the reproduced organism, so much so, in fact, that it cannot be maintained that they constitute the same system. Given that reproduction may involve phenomena like embryogenesis, development and even the possible simultaneous presence of the reproducer and reproduced system - objectors might argue - such causal and phenomenological discontinuities prevent us from considering these systems as instances of the same self-maintaining system. Only individual organisms are genuine self-maintaining systems.

In our view, this objection is wrong, since it is based on an insufficient understanding of the conditions under which an organization can be considered self-maintaining. The crucial requirement, as discussed above, is the causal transmission of the organizational properties. Two selfmaintaining systems on a shorter temporal scale may be said to constitute two temporal instances of a single self-maintaining organization on a larger time scale, if it can be shown that self-maintenance has been causally transmitted from one instance to the other by the maintenance of a material continuity between them. Has the self-maintenance of one instance resulted in the self-maintenance of the other instance or, to put it another way, has the subsequent instance inherited its self-maintaining organization from the previous instance? Since the answer to these questions is, in a fundamental sense, affirmative for the case of the relationship between the reproducer and the reproduced system, we claim that the encompassing organization including them is itself a self-maintaining organization which maintains itself also through reproduction.

As Griesemer has pointed out, the reproduction process does involve the material connection between the reproducer and reproduced system:

"Reproduction (...) is the multiplication of entities with a material overlap of parts between parents and offspring. Material overlap means that parts of the parents (at some time) become parts of the offspring (at some other time). Thus reproduction is no mere transmission or copying of form -- it is a flow of matter" (Griesemer, 2002: 105, emphasis $\left.\operatorname{added}^{\mathrm{xi}}\right)$.

In fact, this means that what happens in this case is the realization of a FFE recursive organization, which displays a spatial proliferation of its core recursive cycle. And this is explained ultimately in terms of the time relation between the production and decay of the constitutive components in a FFE recursive organization. If the rate of replacement of the constitutive components is faster than its decay, the autopoiesis of the system will prompt it to establish reproductive cycles: the system will grow and reproduce; otherwise, it will disintegrate. Only in the very unlikely case of coincidence between the rates of replacement and decay will the autopoiesis 
of the system realize self-maintenance without reproduction (Zepik et al. 2001).

Thus, in a prebiotic chemical scenario, self-reproduction is simply the most frequent form of self-maintenance. When, in a further step, reproduction takes place through genetic mechanisms, it still remains a dynamical FFE cyclic process: template-like reproduction cannot take place without catalysts: it occurs within a "metabolic" autocatalytic cycle (SM organization). As Eschenmosser (2007) has pointed out, both forms of (self)re-production (be they "genetic" or "metabolic", i.e., due to a templatelike structure or to an autocatalytic reaction cycle) are intrinsically cyclic processes. In other words, reproduction is a specific (but far more frequent) form of self-maintenance or self-production (autopoiesis). And this fundamental fact is what grounds Griesemer's remarks on the strong material embeddedness of reproductive dynamics within the metabolic dynamics.

The macroscopic transition produced by the reproductive process can then be seen as the way in which the organization actually manages to selfmaintain beyond the temporal boundaries of individual organisms. Just as the various temporal instances of an individual organism are considered, despite changes and modifications, as a single self-maintaining organization to the extent that the organizational properties are causally transmitted through a material connection across the various instances, so too are the various instances of the inter-generational organization considered as a single self-maintaining organization due to the causal transmission across the instances. The operation is exactly the same, the difference lies only in the level of temporal zoom through which self-maintenance is observed.

This is why development is an essential feature of the self-maintaining organization of living organisms. Once we see reproduction as a process materially connecting the reproducer and the reproduced organizations, development appears as a necessary step in this continuous process of complex self-maintenance. Indeed, self-maintenance of biological individuals cannot be ensured but through a continuous unfolding of changes including reproduction and development.

From this perspective, reproduction is functional because it stems from a means of self-maintenance of an organization. Understanding the reproductive role of a biological trait only as a causal connection between different systems leads us to understand reproduction simply in terms of its contribution to the self-maintenance of a particular type of organization, such as a species or population. This would lead us to the problem related to these kinds of "type-like" organizations mentioned in the section 3.2. (i.e., species, lineages and so on). In fact, reproduction is functional, and the cross-generation traits have functions, because they are related to the selfmaintenance of a FFE token organization ${ }^{\text {xii }}$, not because they involve a "formal" causal connection between a reproducer and a reproduced system. The organizational properties of reproducer and reproduced are linked not only by a mere "formal" relationship, but also by a material causation showing that both reproducer and reproduced are parts of the same FFE self-maintaining organization. Since the only relevant ground for functional 
attributions is organizational self-maintenance, all other criteria of distinction between biological systems seem irrelevant for this matter. This is why reproductive traits can be said to be subject to organizational closure and why, then, we ascribe functions to them.

\section{Conclusions}

The aim of this paper was to provide a solution for an ontological problem which the organizational account has to resolve, i.e. the need to account for intra-generation and cross-generation functions without referring to different natural kinds, and avoiding the splitting account proposed by Delancey. Both intra- and cross-generation functions can be defined as causal effects of a trait subject to organizational closure in self-maintaining systems, since the characterization of an organizational self-maintaining system applies to both kinds of systems which ground the two classes of functions.

It should be emphasized that this solution to the ontological problem stems from the specific version of the organizational account that we propose, which appeals to the idea of self-maintaining organizations as the relevant ground for the naturalization of biological functions. Indeed, at least two implications of our proposal for the philosophical debate should be mentioned.

On the one hand, our account integrates and justifies Schlosser's and McLaughlin's accounts, by showing why it is legitimate to defend a unified organizational account of both intra- and cross-generation biological functions. These accounts are open to Delancey's criticism because, by defining functions as the self-reproduction of traits within complex systems, they have to acknowledge the distinction between the self-sustenance of an instance within an individual system and the self-reproduction of a new instance of the same type in a different system. If the definition is restricted to the self-reproduction of the trait, the account cannot avoid distinguishing between the two cases, and has to split the definition. Our account corrects Schlosser's one in two crucial aspects. Firstly, it defines functions not as causal contributions to the self-reproduction of traits, but rather as contributions of traits, subject to organizational closure, to the selfmaintenance of a system. Secondly, it identifies organizational selfmaintaining systems, and not generically complex systems, as the relevant class of systems that grounds functional ascriptions.

On the other hand, although there is a close relationship between the notion of organizational self-maintenance and that of autonomy used by Christensen and Bickhard, it seems to us that the former is a better candidate as a naturalized grounding for (both intra- and cross-generation) biological functions. By focusing on organizational self-maintenance, our account provides a theoretical justification for the idea that it is the same organization which self-maintains through the reproduction transition. Just as we admit that the various instances of a trait within an individual organism can be considered as the same token in spite of continuous changes, we can admit that traits bearing cross-generation functions in the reproducer and reproduced organisms can be said to be the same tokens (as parts of the same organization), when using (and, possibly, only when 
using) organizational self-maintenance as a criterion of identification.

We conclude that our account succeeds in dealing with Delancey's challenge, since it explains how we are to identify organizations that ground cross-generation functions without reference to individual organisms. As a consequence, we suggest applying Ockham's razor to this debate, abandoning a splitting account, and maintaining a unified definition of functions from an organizational perspective.

\section{References}

Bickhard, M.H. (2000). Autonomy, Function, and Representation. Communication and Cognition - Artificial Intelligence, 17(3-4), 111-131.

Bickhard, M.H. (2004). Process and Emergence: Normative Function and Representation. Axiomathes - An International Journal in Ontology and Cognitive Systems, 14, 121-155.

Bigelow, J., \& Pargetter R. (1987). Functions. Journal of Philosophy, 84, 181-196.

Boorse, C. (1976). Wright on Functions. Philosophical Review, 85, 70-86.

Chandresekhar, S. (1961). Hydrodynamic and hydromagnetic stability. Oxford, Clarendon.

Christensen, W.D., \& Bickhard, M.H. (2002). The Process Dynamics of Normative Function. The Monist, 85, 1, 3-28.

Cornish-Bowden, A. Cárdenas, M.L., Letelier J.C. \& Soto-Andrade, J. (2007). Beyond reductionism: metabolic circularity as a guiding vision for a real biology of systems. Proteomics, 7, 839-845.

Cummins, R. (1975). Functional analysis. Journal of Philosophy, 72, 741765. Reprinted in Buller, D.J. (1999). Function, Selection, and Design. SUNY Press. Albany, New York, 57-83.

Collier, J. (1999). Autonomy and process closure as the basis for functionality. In G. Chandler \& J. L. R. van de Vijver (Eds.) Closure: Emergent Organizations and their Dynamics. Volume 901 of the New York Academy of Sciences.

Davies, P.S. (2001). Norms of Nature. Naturalism and the Nature of Functions. Cambridge: MIT Press.

Delancey, C. (2006). Ontology and teleofunctions: A defense and revision of the systematic account of teleological explanation. Synthese, 150, 69-98.

Edin, B. (2008) Assigning biological functions: making sense of causal chains. Synthese, 161, 203-218.

Eschenmosser, A. (2007) The search for the chemistry of life's origin. Tetrahedron 63: 12821-12844.

Field, R. J., Körös, E. \& Noyes, R. M. (1972). Oscillations in Chemical Systems. II. Thorough Analysis of Temporal Oscillation in the BromateCerium-Malonic Acid System". Journal of the American Chemical Society, 94, 8649-8664. 
Field, R. J. \& Noyes, R. M. (1974). Oscillations in chemical systems. IV. Limit cycle behavior in a model of a real chemical reaction. Journal of Chemical Physics 60, 1877-1884.

Gánti, T. (1975). Organisation of chemical reactions into dividing and metabolizing units: The chemotons. BioSystems 7, 15-21.

Glansdorff, P. \& Prigogine, I. (1971). Thermodynamics of structure, stability and fluctuations. Wiley, London.

Godfrey-Smith, P. (1994). A modern history theory of functions. Noûs, 28, 344-362.

Godfrey-Smith, P. (2009). Darwinian Populations and Natural Selection. Oxford University Press, Oxford.

Griesemer, J.R. (2000). The Units of Evolutionary Transition. Selection 1: 67-80.

Griesemer, J.R. (2002). What Is "Epi" about Epigenetics? In Van Speybroeck, L., Van de Vijver, G. and De Waele, D. (Eds). From Epigenesis to Epigenetics: the Genome in Context. New York Academy of Sciences, New York, 97-110.

Hofmeyr, H-J.S. (2007) The Biochemical factory that autonomously fabricates itself: a systems biological view of the living cell. In Boogerd, F., Bruggeman, F. J., Hofmeyr, J-H. S. \& Westerhoff, H.V. (Eds.) Systems Biology: Philosophical Foundations. Elsevier, Amsterdam, 217-242.

Kauffman, S. (2000). Investigations. Oxford University Press, Oxford.

Luisi, P.L. (1998). About various definitions of life. Origins Life Evol. Biosph. 28, 613-622.

Luisi, P.L. (2006) The Emergence of Life: From Chemical Origins to Synthetic Biology, Cambridge University Press, Cambridge.

Maturana, H., \& Varela, F.J. (1973). De máquinas y seres Vivos - Una teoría sobre la organización biológica. Santiago de Chile: Editorial Universitaria S.A.

McLaughlin, P. (2001). What Functions Explain. Functional Explanation and Self-Reproducing Systems. Cambridge University Press, Cambridge.

Millikan, R.G. (1984). Language, Thought, and Other Biological Categories. MIT Press, Cambridge.

Millikan, R.G. (1989). In defense of proper functions. Philosophy of Science, 56, 288-302.

Moreno, A. \& Ruiz-Mirazo, K. (2009). The problem of the emergence of functional diversity in prebiotic evolution. Biology and Philosophy 24, 5, 585-605.

Mossio, M., Saborido, C., Moreno, A. (2009) An Organizational Account for Biological Functions. British Journal for the Philosophy of Science, 60 (4), 813-841.

Neander, K. (1980). Teleology in Biology. Paper presented to the AAP conference. 
Neander, K. (1991). Function as selected effects: The conceptual analyst's defense. Philosophy of Science, 58, 168-184.

Nicolis, G., \& Prigogine, I. (1977). Self-Organisation in Non-Equilibrium Systems: From Dissipative Structures to Order Through Fluctuation, Wiley, New York.

Pattee, H.H., (1973), The Physical Basis and Origin of Hierarchical Control. In: Pattee, H.H. (Ed.). Hierarchy Theory, Braziller, New York, 73-108.

Pattee, H. H. (1982). Cell psychology: An evolutionary approach to the symbol-matter problem. Cognition and Brain Theory, 4, 325-341.

Rosen, R. (1973): On the dynamical realizations of (M, R)-systems. Bulletin of Mathematical Biophysics 35, 1-9.

Rosen, R. (1991): Life itself: A comprehensive inquiry into the nature, origin and fabrication of life. Columbia Univ. Press, New York.

Ruiz-Mirazo, K. (2001). Physical conditions for the appearance of autonomous systems with open-ended evolutionary capacities. $\mathrm{PhD}$ dissertation, University of the Basque Country.

Ruiz-Mirazo, K. \& Moreno, A. Autonomy as a bridge between the living and cognitive domains. Synthese, forthcoming.

Schlosser, G. (1998). Self-re-production and functionality: A systemstheoretical approach to teleological explanation. Synthese, 116, 303-354.

Varela, F.J. (1979). Principles of Biological Autonomy. New York: North Holland.

Varela, F., Maturana, H. \& Uribe, R (1974). Autopoiesis: The Organisation of Living Systems, its characterization and a model., BioSystems 5: 187196.Wright, L. (1973). Functions. Philosophical Review, 82, 139-168.

Zepik, H., Blöchliger, E. \& Luisi. P.L. (2001) A Chemical Model of Homeostasis Angew. Chemie Int. Ed. 40 No.1: 199-202.

\footnotetext{
i This view of biological systems was initially proposed by pioneers like Rosen (1973; 1991), Maturana (1973), Varela (1974, 1979), Pattee (1973, 1982) and Ganti (1975). Today, this approach is now increasingly accepted, and has been developed in various scientific fields, including theoretical biology (Kauffman, 2000), biochemistry (Luisi, 1998, 2006; Cornish-Bowden at al., 2007), and synthetic biology (Hofmeyr, 2007).

ii See section 3.1 for a more detailed analysis of the organisational account of teleology and normativity.

iii The label "organizational" seems to us to be preferable to, in particular, "systematic" (Delancey) and "systems-theoretical" (Schlosser, even if he refers also to his own approach as an "organizational account"), which may generate confusion with the classical "systemic" approaches, such as those adopted by Cummins (1975) or Davies (2001).

iv According to Schlosser, functional attributions are meaningful only in a specified period of time (p. 318). The second line of the formula means that, taking $t_{0}$ as the beginning of the considered period $T, t_{0}$ precedes $t$, which precedes $t+x+y$ which, in turn, precedes the end of $\mathrm{T}$ (expressed by $\left.\mathrm{t}_{0}+\mathrm{T}\right)$.

v Schlosser emphasize that functional ascriptions necessarily refer to trait types: "The concept of self-re-production is meaningful only if statements (1) and (2) of a function ascription are conceived as statements about types. An individual state or trait does not re-
} 
produce itself per definition. When we talk about recurrence or re-production of a certain state $\mathrm{X}$, we do not claim that an individual state $\mathrm{X} 1$ (t1) is re-established at a later time t2; we only claim that another state of the same type, e.g., X2 (t2), is present at $\mathrm{t} 2$. As a consequence, a function can be ascribed to an individual X1 (t1) only insofar as it can be treated as a token of a type $\mathrm{X}(\mathrm{t})$. Accordingly, there can be no function ascription that applies only to a single token of a type." (Schlosser, 1998: 316-317).

vi Although Delancey does not explicitly mention him, his criticism holds also for McLaughlin's view which, as we specify in the previous section, shares with Schlosser the idea that the notion of "trait self-reproduction" can be applied to the same trait token in a given system, as well as to another trait token of the same type in a different system.

vii The criterion is intrinsic, since not imposed by an external observer, according to some extrinsic reason, and naturalized, since related to a factual feature of the system's nature, and not deduced from some metaphysical or moral principle.

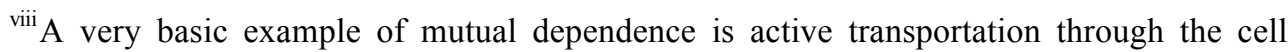
membrane. The level of chemical concentrations that the membrane's active transport retains within the cell is necessary for some metabolic reactions to maintain the appropriate rate to sustain the network of reactions, which in turn produces the membrane, and so on in a circular and interdependent manner.

ix This definition differs slightly in terminology from that proposed in Mossio et al. (2009).

$x$ The idea of autonomy usually has a stronger sense than that of self-maintaining organization since it is supposed to capture all relevant properties of living systems (RuizMirazo \& Moreno, forthcoming; Varela, 1979). In this sense, if an agreement could be reached regarding taking self-maintaining organization as a necessary and sufficient grounding for biological functions, it might be preferable to reserve the label "autonomous systems" for the actual biological instances of a self-maintaining system, i.e. individual organisms

${ }^{x i}$ This point has been the object of an important debate in the philosophy of biology. In the context of Darwinian's theory of evolution, Griesemer's account has been criticized by Godfrey-Smith (2009, pp 79-86) who argues that certain viruses show a purely formal way of reproduction. However, Godfrey-Smith's example is not relevant for our case, since his claim that there is not necessarily a material connection between reproducer and reproduced applies only to molecules (or, at most to aggregates of molecules), not to FFE selfmaintaining organizations, which imply a gathering together of different reactions, i.e., embedding the processes of synthesis of new structures --and degradation of other ones-- in a recursively viable reaction network. Furthermore, if we consider only structural (not organizational) replicators, there would be no functional domain (Moreno \& Ruiz-Mirazo, 2009).

xii Although criteria exist which hold that separate organisms are different tokens and similar types, we believe that the reproductive cycle, as such, is a material instance of a specific FFE self-maintaining organization, and hence is itself a token (although a different token from self-bounded organisms). This is the criterion we use to say that crossgeneration traits are functional within the framework of a self-maintaining token organization. 